\title{
Integrated Arbovirus Surveillance Improves the Detection Onset of Zika Virus in Panama
}

\author{
Gilberto A. Eskildsen, ${ }^{1,2}$ Laura D. Kramer, ${ }^{3}$ Steven D. Zink, ${ }^{3}$ Alan P. Dupuis II, ${ }^{3}$ Susan J. Wong, ${ }^{3}$ Andrea Furuya, ${ }^{3}$ and \\ Jose R. Loaiza ${ }^{1,4,5 *}$ \\ ${ }^{1}$ Instituto de Investigaciones Científicas y Servicios de Alta Tecnología, Panamá, República de Panamá; ${ }^{2}$ Department of Biotechnology, Acharya \\ Nagarjuna University, Guntur, India; ${ }^{3}$ New York State Department of Health, The Wadsworth Center, Albany, New York; ${ }^{4}$ Smithsonian Tropical \\ Research Institute, Balboa Ancon, Republic of Panama; ${ }^{5}$ Programa Centroamericano de Maestría en Entomología, Universidad de Panamá, \\ Panamá, Republic of Panama
}

Abstract. We tested 700 serum samples collected throughout Panama from 2015 to 2016 for detecting antibodies and RNA of arboviruses. In convalescent specimens, microsphere immunoassay detected an antibody prevalence of $59.3 \%$ for dengue virus (DENV) and $30.3 \%$ for Zika virus (ZIKV), which included samples that were collected before the Panamanian surveillance system reported the first case of Zika in the country. For acute sera, the most common arbovirus was DENV with 18 positive samples (6\%), followed by four (1.3\%) of ZIKV and one (0.6\%) of chikungunya virus (CHIKV). Our results indicate a change in the chronology of when ZIKV was first detected in Panama and stress the importance of integrating various approaches to enable improved surveillance of both endemic and emerging arboviruses.

The arthropod-borne virus (e.g., arbovirus) surveillance system at the Panamanian Ministry of Health (MINSA) detected the first case of dengue virus (DENV) in 1993. ${ }^{1}$ Dengue virus was the only arbovirus of epidemiological importance reported in Panama for the next 20 years. Recently, the epidemiology of arboviral diseases changed in the country because of the incursion of pandemic emerging pathogens, such as chikungunya virus (CHIKV) and Zika virus (ZIKV). The first autochthonous case of CHIKV in Panama was reported in August 2014. ${ }^{2,3}$ Zika virus was first detected in Guna Yala Comarca, eastern Panama, in November 2015. ${ }^{4}$ Phylogenetic analysis with partial sequences of nonstructural protein 1 (NS1) revealed similarity to the Asian lineage of ZIKV, which at the time was co-circulating in other countries of Central and South America. ${ }^{5}$ In addition, other endemic arboviruses had also been sporadically reported either from mosquito or from human samples in Panama, including yellow fever virus (YFV) and Mayaro virus (MAYV) ${ }^{6,7}$; yet, no diagnostic scheme or surveillance efforts are implemented currently to test for these pathogens in the country.

The risk for concurrent outbreaks of emerging CHIKV and ZIKV plus additional endemic DENV, YFV, and MAYV complicated clinical diagnosis and disease control in Panama, prompting the evaluation of integrated and new serological and molecular approaches to survey for these arboviruses. Herein, we test acute and convalescent febrile human samples for the presence of RNA and/or antibodies to DENV, ZIKV, CHIKV, and MAYV that were collected in Panama between 2015 and 2016, as a prospective surveillance study. A total of 700 serum samples were divided into two testing groups: 300 acute ( $<6$ days of onset symptoms) samples were tested by molecular multiplex quantitative real-time polymerase chain reaction (qRT-PCR) for RNA amplification and 400 ( $\geq 7$ days of onset symptoms) convalescent sera were assayed with the microsphere immunofluorescence assay (MIA) and plaque reduction neutralization test (PRNT) for detection of antibodies (Supplemental Figure 1). All the samples were collected from human subjects who had not traveled outside Panama in the prior 2 months.

* Address correspondence to Jose R. Loaiza, INDICASAT AIP, 208 City of Knowledge, Panama 0843-01103, Republic of Panama. E-mail: jloaiza@indicasat.org.pa
Our goal was to describe the outcomes of arbovirus surveillance in Panama using serologic techniques, such as MIA and PRNT, and molecular approaches to detect the presence of viral RNA using multiplex qRT-PCR. The serologic assays can discriminate between DENV and ZIKV infections by detecting both structural and nonstructural protein antibodies in human samples, particularly during primary infection. ${ }^{8}$ Serum samples were collected for surveillance purposes through the National Dengue Surveillance Program at MINSA. The sample collection protocol was approved by MINSA's coordination office for regulation and health research before further shipping of the samples to the Griffin Laboratory at the Wadsworth Center, New York State Department of Health, Albany, NY. Quantitative real-time polymerase chain reaction to detect CHIKV and ZIKV RNA in acute samples, plus a set of probes for detecting DENV 1/3 and DENV 2/4 serotypes, was implemented. RNA extraction was carried out using the MagMAXTM-96 Viral RNA Isolation Kit (Ambion, Carlsbad, CA) following the manufacturer's instructions. Primers and probes were designed based on previous studies. ${ }^{9-11}$ Furthermore, to differentiate between CHIKV and ZIKV infections with the convalescent sample set, we used $\mathrm{MIA}^{8}$ with seven different recombinant antigens (ZIKV E, ZIKV NS1, ZIKV NS5, DENV-1 NS1, DENV-2 NS1, DENV-3 NS1, and DENV-4 NS1), conjugated to microsphere beads and mixed with patient serum. Results are reported as median fluorescent intensity (MFI). Avidity assay was further performed on reactive specimens using a modification of the original protocol of $\mathrm{MIA},{ }^{12}$ which includes an extra incubation step with urea. To assess whether a clinical sample contains low, medium, or high avidity antibodies, we calculated the avidity index of each sample expressed as a percentage, i.e., the ratio of MFI values in wells treated with urea to MFI values in wells treated with blocking/storage buffer (phosphate buffered saline containing $0.05 \%$ sodium azide), multiplied by $100 .{ }^{13}$ In addition, convalescent samples were screened at 1:20 dilution by PRNT to assess neutralizing antibodies against CHIKV and MAYV. The virus used in the PRNT assay was CHIKV strain 91077 (i.e., human isolate, India, 2006) and MAYV strain TRVL-4675 (i.e., human isolate, Trinidad, 1954).

The majority of febrile patients had headache $(88 \%)$, chills (84\%), arthralgia (81\%), myalgia (79\%), and rash (71\%); less frequently retro-orbital pain (38) and vomiting (39\%); and 
sometimes diarrhea (18\%) and conjunctivitis (15\%). Quantitative real-time polymerase chain reaction testing detected viral RNA in 24 of 300 acute samples for one or more arboviruses. The most common arbovirus was DENV with 18 positive samples $(6 \%)$ encompassing 10 DENV serotype 1 or 3 and 8 DENV serotype 2 or 4 , followed by 4 positive samples (1.3\%) for ZIKV and $1(0.6 \%)$ for CHIKV (Table 1). One sample tested positive for the RNA of both DENV and ZIKV, indicating coinfection. Positive samples in the qRT-PCR testing presented a threshold cycle (Ct values) ranging from 31 to 37 . Interestingly, one of the ZIKV qRT-PCRpositive samples was collected in Panama City in July 2015, 4 months before the epidemiological surveillance system reported the first outbreak in Guna Yala, ${ }^{5}$ indicating a different chronology for the first detection of Zika in Panama. Possibly, an infected person traveled from Guna Yala to Panama City at that time, but regardless, it precedes the first outbreak in Guna Yala.

All the convalescent samples tested for the presence of antibodies against CHIKV and MAYV with the PRNT were negative, that is, < 1:20. Furthermore, roughly $237(59.3 \%)$ and $121(30.3 \%)$ sera showed evidence of antibodies to DENV and ZIKV, respectively, with the MIA assay, which means that patients had been exposed to both arboviruses. The avidity test to distinguish between past (i.e., likely DENV) and recent infections (i.e., likely ZIKV) indicated that most ZIKV infections occurred recently, whereas DENV infections occurred in both forms, recently and in the past. In addition, two samples collected in the Guna Yala Comarca, which is the location of the first outbreak of ZIKV in June 2015, had low avidity antibodies against ZIKV, indicating that these patients had a recent infection. Molecular findings further confirm that ZIKV was perhaps circulating in Panama before November 2015, when the Panamanian surveillance system first detected the virus.

Historically, PRNT serological testing and viral cell culture were used in Panama to survey for DENV through the centralized National Reference Laboratory at the Gorgas Memorial Institute for Health Studies (ICGES). In 2003, the ICGES established a conventional PCR protocol (singleplex) for the confirmation of DENV infections. Then, after 2009, MINSA implemented nationwide serological confirmation of DENV with a specific enzyme-linked immunosorbent assay (ELISA). However, no discriminatory surveillance approach was available for CHIKV and ZIKV at the time they first entered Panama. Here, we integrate arbovirus disease surveillance approaches to show that combining molecular and serological tools allows detection of DENV and ZIKV in both acute and convalescent

TABLE 1

Summary of the results of arbovirus surveillance using molecular and serological techniques on 700 sera

\begin{tabular}{lcr}
\hline & Results & $\%$ \\
\hline Quantitative real-time polymerase chain reaction (acute samples) \\
DENV+ & 18 & 6.0 \\
ZlKV+ & 4 & 1.3 \\
Chikungunya virus+ & 1 & 0.3 \\
DENV-ZIKV+ & 1 & 0.3 \\
Negatives & 276 & 92.0 \\
Microsphere immunofluorescence assay (convalescent samples) \\
DENV reactive & 237 & 59.3 \\
ZlKV reactive & 121 & 30.3 \\
Nonreactive and indeterminate & 163 & 40.7
\end{tabular}

DENV = dengue virus; ZIKV = Zika virus. Three hundred acute samples: presented on days $1-6$, where day 1 was defined as the day post-onset symptoms. Four hundred convalescent samples: presented on day 7 or more post-onset symptoms. samples. For example, multiplex qRT-PCR not only allows differentiation among DENV, CHKV, and ZIKV infections plus coinfections with acute samples all at once ${ }^{11,14,15}$ but also permits detection of positive sera even when viremia is undetectably low. A recent study by Waggoner et al. (2016) ${ }^{16}$ in Nicaragua depicted a $20.5 \%$ arbovirus coinfection rate with a similar multiplex qRT-PCR protocol to that used here, which is much higher than what we report from Panama. Therefore, we encourage future sero-surveillance studies with more samples from across the entire country to track the spread of both CHKV and ZIKV.

Conventional ELISA diagnosis of DENV and ZIKV is complicated because of cross-reactivity among members of the genus Flavivirus. For example, interpretation of the current IgMcapture ELISAs for distinguishing between ZIKV and DENV is challenging because of the cross-reactivity of antibodies, which require PRNT testing for confirmation. The recently developed MIA/avidity technique allowed us to differentiate which Flavivirus was the more recent infection and sometimes resolved cross-reactivity between DENV and ZIKV in convalescent samples. $^{8}$ This is especially valuable in dengue-endemic countries like Panama, where a significant portion of the human population is likely to harbor DENV antibodies from past infections. Given MINSA's limitations to test acute samples from remote areas of Panama for arboviral infection with qRTPCR, we suggest using paired sera (acute and convalescent) and using PRNT or MIA to distinguish ZIKV and DENV infection, depending on the availability of instruments required to implement these techniques. Whereas it takes between 5 and 7 days to perform PRNT against several viruses simultaneously, the MIA can be performed in a day, even with an avidity assay. In addition, PRNT requires a biosafety level 3 laboratory if $\mathrm{CHIKV}$ is included in the assay. It requires maintenance of cell line, plasticware to culture the cells, and training on conduct of assay and interpretation of results.

We set back the timing for the detection of ZIKV in Panama to June 2015 and suggest that it may have been circulating in Panama City before MINSA reported the post-onset of cases from the Guna Yala Comarca, in November 2015. Altogether, findings indicate a change in the chronology of the first ZIKV detection in Panama and stress the importance of integrating various approaches to enable improved surveillance of endemic and emerging arboviruses.

Received April 24, 2019. Accepted for publication October 2, 2019.

Published online March 30, 2020.

Note: Supplemental table and figure appear at www.ajtmh.org.

Acknowledgments: We thank members of the Department of Epidemiology at MINSA for providing the serum samples used in this study. We appreciate the logistical support from MINSA's members Itza Barahona de Mosca and Lizbeth Cerezo and the academic input of Evelia Quiroz about the history of dengue disease epidemiology in Panama.

Financial support: This work was supported by the National Secretariat for Science, Technology and Innovation of Panama grant (SENACYT-IDDS15-047) to J. R. L. and the travel fellowship grant (SENACYT-APY-GC-2014-024) to G. A. E. Research by J. R. L. is also supported by the National Investigation System of SENACYT (SNI, 05-2016 and 157-2017).

Disclosure: S. J. W. has a patent (U.S. Patent 10,317,413) issued.

Authors' addresses: Gilberto A. Eskildsen, Instituto de Investigaciones Científicas y Servicios de Alta Tecnología, Panamá, Republic of 
Panama, and Department of Biotechnology, Acharya Nagarjuna University, Guntur, India, E-mail: geskildsen@indicasat.org.pa. Laura D. Kramer, Steven D. Zink, Alan P. Dupuis II, Susan J. Wong, and Andrea Furuya, New York State Department of Health, The Wadsworth Center, Albany, NY, E-mails: laura.kramer@health.ny.gov, steven.zink@ health.ny.gov, alan.dupuis@health.ny.gov, susan.wong@health.ny.gov, and andrea.furuya@health.ny.gov. Jose R. Loaiza, Instituto de Investigaciones Científicas y Servicios de Alta Tecnología, Panamá, Republic of Panama, Smithsonian Tropical Research Institute, Balboa Ancon, Republic of Panama, and Programa Centroamericano de Maestría en Entomología, Universidad de Panamá, Panamá, Republic of Panama, E-mail: jloaiza@indicasat.org.pa.

\section{REFERENCES}

1. Quiroz E, Ortega M, Guzmán MG, Vázquez S, Pelegrino JL, Campos C, Bayard V, Vázquez M, Kourí G, 1997. Dengue in Panama, 1993 [article in Spanish]. Rev Cubana Med Trop 49: 86-93.

2. MINSA, 2019. Epidemiología Boletines Semanales | Panama. Panamá, Republic of Panama: Ministerio de Salud. Available at: http://www.minsa.gob.pa/informacion-salud/boletinessemanales-2019. Accessed July 21, 2019.

3. Díaz $Y$ et al., 2015. Chikungunya virus infection: first detection of imported and autochthonous cases in Panama. Am J Trop Med Hyg 92: 482-485.

4. López-Vergès S, Pachar MR, Arauz D, Suárez JA, Moreno B, Gundacker ND, Araúz AB, Suárez M, 2018. Zika virusassociated cerebellitis with complete clinical recovery. Am J Trop Med Hyg 99: 1318-1320.

5. Arauz D et al., 2016. Febrile or exanthematous illness associated with Zika, dengue, and chikungunya viruses, Panama. Emerg Infect Dis 22: 1515-1517.

6. Young NA, Dammin GJ, Johnson KM, Peralta PH, Shelokov A, 1968. Recovery of Venezuelan equine encephalomyelitis virus in Panamá. Am J Trop Med Hyg 17: 432-440.

7. Watts DM, Shope RE, Barrett ADT, Tesh RB, Saeed MF, Nunes M, Wang H, Weaver SC, Vasconcelos PFC, 2000. Nucleotide sequences and phylogeny of the nucleocapsid gene of Oropouche virus. J Gen Virol 81: 743-748.

8. Wong SJ, Furuya A, Zou J, Xie X, Dupuis AP, Kramer LD, Shi P-Y, 2017. A multiplex microsphere immunoassay for Zika virus diagnosis. EBioMedicine 16: 136-140.

9. Callahan JD et al., 2001. Development and evaluation of serotype- and group-specific fluorogenic reverse transcriptase PCR (TaqMan) assays for dengue virus. J Clin Microbiol 39: 4119-4124.

10. Lanciotti RS, Kosoy OL, Laven JJ, Velez JO, Lambert AJ, Johnson AJ, Stanfield SM, Duffy MR, 2008. Genetic and serologic properties of Zika virus associated with an epidemic, Yap State, Micronesia, 2007. Emerg Infect Dis 14: 1232-1239.

11. Naze F, Le Roux K, Schuffenecker I, Zeller H, Staikowsky F, Grivard P, Michault A, Laurent P, 2009. Simultaneous detection and quantitation of chikungunya, dengue and West Nile viruses by multiplex RT-PCR assays and dengue virus typing using high resolution melting. J Virol Methods 162: 1-7.

12. Furuya AKM, Hunt D, George KS, Dupuis AP, Kramer LD, Shi P-Y, Wong S, 2019. Use of the immunoglobulin $G$ avidity assay to differentiate between recent Zika and past dengue virus infections. Clin Sci (Lond) 133: 859-867.

13. Matheus S, Deparis X, Labeau B, Lelarge J, Morvan J, Dussart P, 2005. Discrimination between primary and secondary dengue virus infection by an immunoglobulin $\mathrm{G}$ avidity test using a single acute-phase serum sample. J Clin Microbiol 43: 2793-2797.

14. Pabbaraju K, Wong S, Gill K, Fonseca K, Tipples GA, Tellier R, 2016. Simultaneous detection of Zika, chikungunya and dengue viruses by a multiplex real-time RT-PCR assay. J Clin Virol 83: $66-71$.

15. Giry C, Roquebert B, Li-Pat-Yuen G, Gasque P, Jaffar-Bandjee $M-C, 2017$. Simultaneous detection of chikungunya virus, dengue virus and human pathogenic Leptospira genomes using a multiplex TaqMan ${ }^{\circledR}$ assay. BMC Microbiol 17: 84.

16. Waggoner JJ et al., 2016. Viremia and clinical presentation in Nicaraguan patients infected with Zika virus, Chikungunya virus, and Dengue virus. Clin Infect Dis 63: 1584-1590. 